Article

\title{
Global Behavior of Certain Nonautonomous Linearizable Three Term Difference Equations
}

\author{
E. J. Janowski and M. R. S. Kulenović *
}

Department of Mathematics, University of Rhode Island, Kingston, RI 02881-0816, USA; ksenada@gmail.com

* Correspondence: mkulenovic@uri.edu

Received: 10 April 2018; Accepted: 7 May 2018; Published: 9 May 2018

\begin{abstract}
We investigate the nonautonomous difference equation with real initial conditions and coefficients $g_{i}, i=0,1$ which are in general functions of $n$ and /or the state variables $x_{n}, x_{n-1}, \ldots$, and satisfy $g_{0}+g_{1}=1$. We also obtain some global results about the behavior of solutions of the nonautonomous non-homogeneous difference equation where $g_{i}, i=0,1,2$ are functions of $n$ and/or the state variables $x_{n}, x_{n-1}, \ldots$, with $g_{0}+g_{1}=1$. Our results are based on the explicit formulas for solutions. We illustrate our results by numerous examples.
\end{abstract}

Keywords: difference equation; identity; linear; nonautonomous; periodic; stability; solution

JEL Classification: AMS 2010 Mathematics Subject Classification: 39A10; 39A20; 39A30

\section{Introduction}

We investigate the global behavior of the second order difference equation

$$
x_{n+1}=g_{0} x_{n}+g_{1} x_{n-1}, \quad n=0,1, \ldots,
$$

where the functions $g_{i}: \mathbb{R}^{k} \rightarrow \mathbb{R}, i=0,1 ; k=1,2, \ldots$, are such that

$$
g_{0}+g_{1}=1, \quad n=0,1, \ldots
$$

Here, the functions $g_{i}, i=0,1$ are in general functions of $n$ and/or the state variables $x_{n}, x_{n-1}, \ldots$ So Equation (1) is indeed a nonautonomous equation. It can also be an equation of order higher than two. As we have shown in [1] if $\left|g_{0}\right|+\left|g_{1}\right| \leq C<1$, then the zero equilibrium of Equation (1) is globally asymptotically stable, and if $\left|g_{0}\right|+\left|g_{1}\right| \leq 1$, then the zero equilbrium of Equation (1) is stable. Furthermore, as we have shown in [1], if $g_{0}+g_{1} \geq r>1$, where $r$ is a constant independent of $n$ and the state variables, then Equation (1) has unbounded solutions. Thus, the most interesting case is when condition (2) is satisfied. This case was addressed in [1] for the general $k$-th order difference equation, and some attractivity results of the equilibrium solutions were given there. However, a restriction that $g_{i} \geq 0, i=0,1$ was imposed. Similar results were obtained in [2] where attractivity results of the periodic solutions were obtained.

When Equation (1) is a linear equation with constant coefficients, condition (2) implies that we have the non-hyperbolic case since $\lambda=1$ would be a characteristic root. In general, difference equations can be very sensitive to any kind of perturbations in the non-hyperbolic case as we can see from the following two examples:

Example 1. Consider the difference equation

$$
x_{n+1}=\frac{1}{n} x_{n}+\frac{n-1}{n} x_{n-1}, \quad n=1,2, \ldots
$$


Starting with the two initial conditions $x_{0}, x_{1}$ we obtain the corresponding solution to be $x_{n}=x_{1}, n \geq 1$. The limiting case of Equation (3), as $n \rightarrow \infty$, is the equation

$$
x_{n+1}=x_{n-1}, \quad n=1,2, \ldots
$$

where every non-constant solution is the period-two solution $\left\{x_{0}, x_{1}\right\}$.

Example 2. Consider the difference equation

$$
x_{n+1}=\left(\frac{1}{2}+\frac{1}{2 n}\right) x_{n}+\left(\frac{1}{2}-\frac{1}{2 n}\right) x_{n-1}, \quad n=1,2, \ldots
$$

Starting with two initial conditions $x_{0}, x_{1}$ we obtain the corresponding solution to be $x_{n}=x_{1}, n \geq 1$. The limiting case of Equation (4), as $n \rightarrow \infty$, is the equation

$$
x_{n+1}=\frac{1}{2} x_{n}+\frac{1}{2} x_{n-1}, \quad n=1,2, \ldots
$$

where every non-constant solution is given as:

$$
x_{n}=\frac{1}{3}\left(x_{0}+2 x_{1}+2\left(x_{0}-x_{1}\right)\left(-\frac{1}{2}\right)^{n}\right), \quad n=0,1, \ldots
$$

Thus the limiting equation has all solutions asymptotic to $\frac{x_{0}+2 x_{1}}{3}$.

Example 3. The following two difference equations

$$
x_{n+1}=\frac{x_{n}^{2}}{a+x_{n}}+\frac{a x_{n-1}}{a+x_{n}}, \quad n=1,2, \ldots
$$

and

$$
x_{n+1}=\frac{b x_{n}}{b+x_{n-1}}+\frac{x_{n-1}^{2}}{b+x_{n-1}}, \quad n=1,2, \ldots
$$

where $a, b>0, x_{0}, x_{1} \geq 0$, which have respective linearizations

$$
x_{n+1}=\frac{x_{n}}{a+x_{n}} x_{n}+\frac{a}{a+x_{n}} x_{n-1}, \quad n=1,2, \ldots
$$

and

$$
x_{n+1}=\frac{b}{a+x_{n-1}} x_{n}+\frac{x_{n-1}}{b+x_{n-1}} x_{n-1}, \quad n=1,2, \ldots,
$$

with the condition (2) satisfied, possible models in population dynamics as all transition functions are Holling type functions, see [3] for further references.

There are many ways that a general second order difference equation of the form

$$
x_{n+1}=f\left(n, x_{n}, x_{n-1}\right), \quad n=0,1, \ldots
$$

where $f$ is a real function from $\mathbb{R}^{3}$ to $\mathbb{R}$, can be transformed into the linearized form (1). See [1,2,4] for linearization methods based on algebraic manipulations and especially [5] for symmetries based methods.

The linear Equation (1) can also be used to obtain generalized identities which can be used to study the behavior of the solutions of the difference equation, see [1,2]. Identities are the building blocks of semicycle analysis, method of invariants and rate of convergence. See [4,6-14]. 
We also extend our investigation to the second order nonautonomous non-homogeneous difference equation

$$
x_{n+1}=g_{0} x_{n}+g_{1} x_{n-1}+g_{2}, \quad n=0,1, \ldots,
$$

where $g_{0}, g_{1}$ and $g_{2}$ are real valued functions which depend on $n$ and satisfy condition (2).

The technique which will be used to obtain our results is based on the explicit formulas for the solutions of Equations (1) and (7). These formulas are of independent interest and can be used in a variety of situations.

We illustrate our results with several examples.

\section{Invariants}

Our first result gives a connection between Equation (1) under the condition (2) and the existence of an invariant.

Lemma 1. Consider Equation (1) under the condition (2) where $g_{0}, g_{1}: \mathbb{R}^{k+1} \rightarrow \mathbb{R}$. Then Equation (1) has an invariant of the form $I_{n}=x_{n}+g_{1} x_{n-1}$ if and only if $g_{1}$ is a constant.

Proof. First, assume condition (2), where $g_{1}=A \in \mathbb{R}$ for $n \geq 0$, is satisfied. Hence Equation (1) becomes

$$
x_{n+1}=(1-A) x_{n}+A x_{n-1}, \quad n \geq 0 .
$$

Thus

$$
x_{n+1}+A x_{n}=x_{n}+A x_{n-1}, \quad n \geq 0
$$

and so

$$
I_{0}=x_{0}+A x_{-1}=x_{n}+A x_{n-1}, \quad n \geq 0
$$

is an invariant for Equation (1).

Second, assume that Equation (1) has an invariant of the form $I_{n}=x_{n}+g_{1} x_{n-1}$. Then the following relation must be satisfied

$$
x_{n+1}+g_{1} x_{n}=x_{n}+g_{1} x_{n-1}, \quad n \geq 0 .
$$

If $g_{1}$ is not a constant for all $n \geq 0$, then $g_{1}=A_{n}$. Thus

$$
x_{n+1}+A_{n+1} x_{n}=I_{n+1}=I_{n}=x_{n}+A_{n} x_{n-1}, \quad n \geq 0 .
$$

Since by condition (2) $g_{0}=1-A_{n}$ for all $n \geq 0$ then Equation (1) becomes

$$
x_{n+1}=\left(1-A_{n}\right) x_{n}+A_{n} x_{n-1}, \quad n \geq 0,
$$

which by substitution in Equation (9) gives

$$
\left(1-A_{n}\right) x_{n}+A_{n} x_{n-1}+A_{n+1} x_{n}=x_{n}+A_{n} x_{n-1}
$$

and so $x_{n}\left(A_{n+1}-A_{n}\right)=0$ for all $n \geq 0$.

Note that the invariant must hold for every solution of Equation (1) including the infinite number of constant solutions $x_{n}=x_{0}, n \geq 0$. Thus $A_{n+1}=A_{n}$ for all $n \geq 0$, so $g_{1}$ is a constant for $n \geq 0$.

Remark 1. The restriction that $g_{0}$ and $g_{1}$ are constants is a severe restriction, yet the obtained invariant can be useful. This invariant will be used to reduce the order of difference Equation (1) resulting in first order linear difference equation which is solvable. See Proposition 1 and Corollary 2. 
Lemma 1 can be extended to any three term difference equation of the form

$$
x_{n+l}=g_{i} x_{n-i}+g_{k} x_{n-k}, \quad n=0,1, \ldots,
$$

where $l \in\{1,2, \ldots\}$ and $i, k \in Z$ such that $-l<i<k$ with

$$
g_{i}+g_{k}=1, \quad n=0,1, \ldots,
$$

Lemma 2. Let $l \in\{1,2, \ldots\}$ and $i, k \in Z$ such that $-l<i<k$. Consider Equation (10) where $g_{i}, g_{k}: \mathbb{R}^{k+l} \rightarrow$ $\mathbb{R}$ and condition (11) is satisfied. Then Equation (10) has an invariant of the form

$$
I_{n}=x_{n+l-1}+\ldots+x_{n-i}+g_{k} x_{n-1-i}+\ldots+g_{k} x_{n-k}, \quad n=0,1, \ldots
$$

if and only if $g_{k}$ is a constant.

Proof. First assume condition (11) and that $g_{k}=A$ is a constant. Then $g_{i}=1-A$ for $n \geq 0$ and so Equation (10) becomes

$$
x_{n+l}=x_{n-i}-A x_{n-i}+A x_{n-k}, \quad n=0,1, \ldots
$$

Then Equation (12) is an invariant. Indeed

$$
\begin{aligned}
I_{n+1} & =x_{n+l}+x_{n+l-1}+\ldots+x_{n+1-i}+A x_{n-i}+\ldots+A x_{n+1-k} \\
& =\left(x_{n-i}-A x_{n-i}+A x_{n-k}\right)+x_{n+l-1}+\ldots+x_{n+1-i}+A x_{n-i}+\ldots+A x_{n+1-k} \\
& =x_{n+l-1}+\ldots+A_{n} x_{n-k}, \quad n=0,1, \ldots
\end{aligned}
$$

Second, assume that Equation (12) is an invariant of Equation (10). If $g_{k}$ is not a constant for all $n \geq$ 0 , then $g_{k}$ is a function of $n$ that is, $g_{k}=A_{n}, n=0,1, \ldots$ Then Equation (10) with condition (11) becomes

$$
x_{n+l}=\left(1-A_{n}\right) x_{n-i}+A x_{n-k}, \quad n=0,1, \ldots
$$

and $I_{n+1}=I_{n}$ implies that for $n \geq 0$

$$
\left(x_{n-i}+x_{n-1-i}+\ldots+x_{n+1-k}\right)\left(A_{n+1}-A_{n}\right)=0 .
$$

Therefore $A_{n+1}=A_{n}$ for all $n \geq 0$, which completes the proof.

\section{Representation of Solutions}

Next we give some basic results on the representation of the solutions of Equation (13). These results have importance of their own and have straight-forward extensions to the difference equations in linear normed spaces.

Lemma 3. Consider the difference equation

$$
x_{n+1}=x_{n}+F_{n}\left(x_{n}-x_{n-1}\right)+D_{n}, \quad n=0,1, \ldots,
$$

where $F_{n}$ and $D_{n}$ are real valued functions for $n=0,1, \ldots$, Then for $n=0,1, \ldots$

(a)

$$
x_{n+1}=x_{n}+\left(x_{0}-x_{-1}\right) \prod_{j=0}^{n} F_{j}+\sum_{k=0}^{n} D_{k} \prod_{i=k+1}^{n} F_{i},
$$

where by definition $\prod_{i=k+1}^{n} F_{i}=1$ when $k+1>n$; 
(b)

$$
x_{n+1}=x_{0}+\left(x_{0}-x_{-1}\right) \sum_{i=0}^{n} \prod_{j=0}^{i} F_{j}+\sum_{i=0}^{n} D_{i}\left(1+\sum_{k=1}^{n-i} \prod_{j=i+1}^{k+i} F_{j}\right),
$$

where by definition $\sum_{k=1}^{n-i} \prod_{j=i+1}^{k+i} F_{j}=0$ when $n<i+1$.

Proof. Set $u_{n}=x_{n}-x_{n-1}, n=0,1, \ldots$, in Equation (13). We obtain:

$$
u_{n+1}=F_{n} u_{n}+D_{n}, \quad n=0,1, \ldots
$$

which is a first order linear nonautonomous non-homogeneous difference equation whose solution is

$$
u_{n}=\prod_{k=0}^{n-1} F_{k} u_{0}+\sum_{k=0}^{n-1} D_{k} \prod_{j=k+1}^{n-1} F_{j}, \quad n=1,2, \ldots
$$

Thus $x_{n}$ satisfies a first order linear nonautonomous non-homogeneous difference equation

$$
x_{n}=x_{n-1}+u_{n}, \quad n=0,1, \ldots,
$$

whose solution is given by Equation (14). By iterating Equation (14) we obtain Equation (15).

From Lemma 3 we can obtain an explicit formula for the solution of the difference Equation (7) where $g_{0}, g_{1}$ and $g_{2}$ are real valued functions which depend on $n$ and satisfy condition (2). For convenience of notation set $g_{0}=A_{n}, g_{1}=B_{n}$ and $g_{2}=D_{n}$ at $n=0,1, \ldots$ Taking into account that $A_{n}+B_{n}=1$ Equation (7) becomes

$$
x_{n+1}=x_{n}-B_{n}\left(x_{n}-x_{n-1}\right)+D_{n}, \quad n=0,1, \ldots .
$$

By Lemma 3 part (b) with $F_{n}=-B_{n}, n \geq 0$ we get that for $n=0,1, \ldots$

$$
x_{n+1}=x_{0}+\left(x_{0}-x_{-1}\right) \sum_{i=0}^{n}(-1)^{i+1} \prod_{j=0}^{i} B_{j}+\sum_{i=0}^{n} D_{i}\left(1+\sum_{k=1}^{n-i}(-1)^{k} \prod_{j=1+i}^{k+i} B_{j}\right)
$$

and so for $n=0,1, \ldots$

$$
x_{n}=\left(1+\sum_{j=0}^{n-1}(-1)^{j+1} \prod_{i=0}^{j} B_{i}\right) x_{0}+\left(\sum_{j=0}^{n-1}(-1)^{j} \prod_{i=0}^{j} B_{i}\right) x_{-1}+\sum_{i=0}^{n-1} D_{i}\left(1+\sum_{k=1}^{n-i-1}(-1)^{k} \prod_{j=1+i}^{k+i} B_{j}\right) .
$$

\section{Main Results}

In this section we present our main results. Consider Equation (7) where the function $g_{2}=D_{n}, n=0,1, \ldots$, in which case Equation (7) becomes

$$
x_{n+1}=g_{0} x_{n}+g_{1} x_{n-1}+D_{n}, \quad n=0,1, \ldots .
$$

Remark 2. Consider Equation (18) where the functions $g_{0}$ and $g_{1}$ satisfies condition (2).

(1) If for some $N=0,1, \ldots D_{N} \neq 0$, then Equation (18) does not have a constant solution. Indeed, assume that Equation (18) has a constant solution $\bar{x}$. Then $D_{N}=\bar{x}-g_{0} \bar{x}-g_{1} \bar{x}=\left(1-g_{0}-g_{1}\right) \bar{x}=0$, which is a contradiction.

(2) If $x_{0}=x_{-1}$ and $D_{n}=0$ for all $n \geq 0$, then for $n \geq 0, x_{n+1}=x_{0}$, which follows from Lemma 3 part (b). 
Theorem 1. Consider Equation (18) where the functions $g_{0}$ and $g_{1}$ satisfies condition (2).

Let $r \in(0, \infty)$ and assume that $x_{n} \in \mathbb{R}$. Assume that either $x_{0} \neq x_{-1}$ or $D_{n} \neq 0$ for some $n \geq 0$.

(1) Suppose that $g_{1} \leq-1$ for $n \geq 0$.

(a) If $x_{0} \geq x_{-1}$ and $D_{n} \geq 0$ for $n \geq 0$, then $\lim _{n \rightarrow \infty} x_{n}=\infty$;

(b) If $x_{0} \leq x_{-1}$ and $D_{n} \leq 0$ for $n \geq 0$, then $\lim _{n \rightarrow \infty} x_{n}=-\infty$;

(2) Suppose that $0<\left|g_{1}\right| \leq r<1$ for $n \geq 0$ and $\sum_{i=0}^{\infty} D_{i}$ converges.

(a) If either $D_{n}=0$ for $n \geq 0$ or $D_{n} \geq 0$ for $n \geq 0$, then every solution of Equation (18) converges to a limit point;

(b) If $D_{n} \leq 0$ for $n \geq 0$, then every solution of Equation (18) is bounded;

(3) Suppose that $g_{1}=0$ for all $n \geq 0$, and let $a \in \mathbb{R}$.

(a) If $D_{n}=0$ for $n \geq 0$, then $x_{n}=x_{0}$;

(b) If $\sum_{i=0}^{\infty} D_{i}$ converges, then every solution of Equation (18) converges to a limit point;

(c) If $\sum_{i=0}^{\infty} D_{i}= \pm \infty$, then $\lim _{n \rightarrow \infty} x_{n}= \pm \infty$;

(d) If $D_{2 i}=a$ and $D_{2 i+1}=-a, i=0,1, \ldots$, then every solution of Equation (18) is a period-two sequence of the form ..., $x_{0}+a, x_{0}, \ldots$.

(4) Suppose that $g_{1}=0$ for finite number of $n \geq 0$

(a) If $D_{n}=0$ for $n \geq 0$, then every solution of Equation (18) is eventually equal to a constant;

(b) If $D_{n} \neq 0$ for some $n \geq 0$, then the solution of Equation (18) may have all kinds of dynamic scenarios which depend on the properties of the nonhomogeneous part.

(5) Suppose that $g_{1}=1$ for $n \geq 0$.

(a) If $D_{n}=0$ for $n \geq 0$, then every solution of Equation (18) is a period-two sequence of the form ..., $x_{-1}, x_{0}, \ldots$;

(b) If either $\sum_{i=0}^{\infty} D_{2 i}= \pm \infty$ or $\sum_{i=0}^{\infty} D_{2 i+1}= \pm \infty$, then every solution of Equation (18) is unbounded;

(c) If $\sum_{i=0}^{\infty} D_{2 i}$ and $\sum_{i=0}^{\infty} D_{2 i+1}$ converge, then every solution of Equation (18) converges to (not necessarily minimal) period-two sequence;

(6) Suppose that $1<r \leq g_{1}$ for $n \geq 0$. Assume that for $n \geq 0$ either

$$
x_{0} \geq x_{-1}, D_{2 n} \leq 0, D_{2 n+1} \geq 0
$$

or

$$
x_{0} \leq x_{-1}, D_{2 n} \geq 0, D_{2 n+1} \leq 0 .
$$

Then every solution of Equation (18) is unbounded.

Proof. When we apply condition (2) to Equation (18) we get that

$$
x_{n+1}=x_{n}-g_{1}\left(x_{n}-x_{n-1}\right)+D_{n}, \quad n=0,1, \ldots
$$

For convenience of notation set $F_{n}=-g_{1}$ and $H_{n}=g_{1}$ at each $n=0,1, \ldots$ For $n \geq 0$ let $R_{n}$ be the sum of positive terms and let $S_{n}$ be the sum of negative terms.

(1) Suppose that $g_{1} \leq-1$ for $n \geq 0$. Then for $n \geq 0, F_{n} \geq 1$. By Lemma 3 part (b) we get that for $n \geq 0$ 


$$
x_{n+1}=x_{0}+\left(x_{0}-x_{-1}\right) \sum_{i=0}^{n} \prod_{j=0}^{i} F_{j}+\sum_{i=0}^{n} D_{i}\left(1+\sum_{k=1}^{n-i} \prod_{j=1+i}^{k+i} F_{j}\right) .
$$

If $D_{n} \neq 0$ for some $n \geq 0$, then let $N$ be the first $n \geq 0$ such that $D_{n} \neq 0$. Then for $n \geq 0$

$$
x_{n+1}=x_{0}+\left(x_{0}-x_{-1}\right) \sum_{i=0}^{n} \prod_{j=0}^{i} F_{j}+D_{N}\left(1+\sum_{k=1}^{n-N} \prod_{j=1+N}^{k+N} F_{j}\right)+\sum_{i=N+1}^{n} D_{i}\left(1+\sum_{k=1}^{n-i} \prod_{j=1+i}^{k+i} F_{j}\right) .
$$

Observe that for $i=0,1, \ldots 1 \leq \prod_{j=0}^{i} F_{j}$ and for $k \geq j, 1 \leq \prod_{j=1+i}^{k+i} F_{j}$. Thus for $n \geq 0 n<\sum_{i=0}^{n} \prod_{j=0}^{i} F_{j}$ and for $n \geq N n-N<1+\sum_{k=1}^{n-N} \prod_{j=1+N}^{k+N} F_{j}$.

(a) Assume that $x_{0} \geq x_{-1}$ and $D_{n} \geq 0, n=0,1, \ldots$. Then for $n \geq N$

$$
\begin{aligned}
x_{n+1} & =x_{0}+\left(x_{0}-x_{-1}\right) \sum_{i=0}^{n} \prod_{j=0}^{i} F_{j}+D_{N}\left(1+\sum_{k=1}^{n-N} \prod_{j=1+N}^{k+N} F_{j}\right)+R_{n} \\
& >x_{0}+\left(x_{0}-x_{-1}\right) n+(n-N) D_{N} .
\end{aligned}
$$

Thus $x_{n} \rightarrow \infty$ as $n \rightarrow \infty$.

(b) Assume that $x_{0} \leq x_{-1}$ and $D_{n} \leq 0, n=0,1, \ldots$ Then for $n \geq N$

$$
\begin{aligned}
x_{n+1} & =x_{0}+\left(x_{0}-x_{-1}\right) \sum_{i=0}^{n} \prod_{j=0}^{i} F_{j}+D_{N}\left(1+\sum_{k=1}^{n-N} \prod_{j=1+N}^{k+N} F_{j}\right)+S_{n} \\
& <x_{0}+\left(x_{0}-x_{-1}\right) n+(n-N) D_{N} .
\end{aligned}
$$

Thus $x_{n} \rightarrow-\infty$ as $n \rightarrow \infty$.

(2) Suppose that $0<\left|g_{1}\right| \leq r<1$ for $n \geq 0$ and $\sum_{i=0}^{\infty} D_{i}$ converges. Then for $n \geq 00<\left|H_{n}\right| \leq r<1$.

By Lemma 3 (b) with $F_{n}=-\left|H_{n}\right|$ we have that for $n \geq 0$

$$
x_{n+1}=x_{0}+\left(x_{0}-x_{-1}\right) \sum_{i=0}^{n}(-1)^{i+1} \prod_{j=0}^{i}\left|H_{j}\right|+\sum_{i=0}^{n} D_{i}\left(1+\sum_{k=1}^{n-i}(-1)^{k} \prod_{j=1+i}^{k+i}\left|H_{j}\right|\right) .
$$

Observe that for $i=0,1, \ldots, 0<\left|(-1)^{i+1} \prod_{j=0}^{i} H_{j}\right| \leq r^{i+1}$. Hence the series $\sum_{i=0}^{n}(-1)^{i+1} \prod_{j=0}^{i} H_{j}$ converges by the comparison test for series to the limiting value.

(a) If $D_{n}=0$ for $n \geq 0$, then $x_{n}$ converges to a limit as $n \rightarrow \infty$. Now assume that $D_{n} \geq 0$ for $n \geq 0$. Observe that for $n \geq 0$ and for any $i=0,1, \ldots$, such that $n \geq i+1$ we have the following:

When $n-i$ is odd, then

$$
\begin{aligned}
0 & \leq 1-\left|H_{i+1}\right|+\left(1-\left|H_{i+3}\right|\right)\left|H_{i+1}\right|\left|H_{i+2}\right|+\ldots+\left(1-\left|H_{n}\right|\right) \prod_{j=1+i}^{n-1}\left|H_{j}\right| \\
& =1+\sum_{k=1}^{n-i}(-1)^{k} \prod_{j=1+i}^{k+i}\left|H_{j}\right|<1+\sum_{k=1}^{n-i} \prod_{j=1+i}^{k+i}\left|H_{j}\right|<\sum_{k=0}^{\infty} r^{k}=\frac{1}{1-r} .
\end{aligned}
$$

When $n-i$ is even, then

$$
\begin{aligned}
0 & \leq 1-\left|H_{i+1}\right|+\left(1-\left|H_{i+3}\right|\right)\left|H_{i+1}\right|\left|H_{i+2}\right|+\ldots+\left(1-\left|H_{n-1}\right|\right) \prod_{j=1+i}^{n-2}\left|H_{j}\right|+\prod_{j=1+i}^{n}\left|H_{j}\right| \\
& =1+\sum_{k=1}^{n-i}(-1)^{k} \prod_{j=1+i}^{k+i}\left|H_{j}\right|<1+\sum_{k=1}^{n-i} \prod_{j=1+i}^{k+i}\left|H_{j}\right|<\sum_{k=0}^{\infty} r^{k}=\frac{1}{1-r} .
\end{aligned}
$$

Hence for $n \geq 0$ and for any $i=0,1, \ldots$, such that $n \geq i+1$ and $D_{i}>0$

$$
0 \leq D_{i}\left(1+\sum_{k=1}^{n-i}(-1)^{k} \prod_{j=1+i}^{k+i}\left|H_{j}\right|\right)<\frac{D_{i}}{1-r}
$$

and so for $n \geq 0$

$$
0 \leq \sum_{i=0}^{n} D_{i}\left(1+\sum_{k=1}^{n-i}(-1)^{k} \prod_{j=1+i}^{k+i}\left|H_{j}\right|\right)<\frac{1}{1-r} \sum_{i=0}^{n} D_{i}
$$


Thus the series

$$
\sum_{i=0}^{n} D_{i}\left(1+\sum_{k=1}^{n-i}(-1)^{k} \prod_{j=1+i}^{k+i}\left|H_{j}\right|\right)
$$

converges by comparison test and so $x_{n}$ converges to a finite limit.

(b) Assume that $D_{n} \leq 0$ for $n \geq 0$. Then there exists $L \in(-\infty, 0)$ such that $\sum_{i=0}^{\infty} D_{i}=L$.

From part (a) we have that for $n \geq i+1, i=0,1, \ldots$,

$$
0 \leq 1+\sum_{k=1}^{n-i}(-1)^{k} \prod_{j=1+i}^{k+i}\left|H_{j}\right|<\frac{1}{1-r}
$$

Then for $n \geq i+1, i=0,1, \ldots$, and $D_{i}<0$

$$
0 \geq D_{i}\left(1+\sum_{k=1}^{n-i}(-1)^{k} \prod_{j=1+i}^{k+i}\left|H_{j}\right|\right)>\frac{D_{i}}{1-r}
$$

and so

$$
0 \geq \sum_{i=0}^{n} D_{i}\left(1+\sum_{k=1}^{n-i}(-1)^{k} \prod_{j=1+i}^{k+i}\left|H_{j}\right|\right)>\frac{1}{1-r} \sum_{i=0}^{n} D_{i}>\frac{1}{1-r} \sum_{i=0}^{\infty} D_{i}=\frac{L}{1-r} .
$$

If $x_{0}=x_{-1}$, then

$$
x_{0} \geq x_{0}+\sum_{i=0}^{n} D_{i}\left(1+\sum_{k=1}^{n-i}(-1)^{k} \prod_{j=1+i}^{k+i}\left|H_{j}\right|\right)>x_{0}+\frac{L}{1-r} .
$$

Thus $x_{0} \geq x_{n+1}>x_{0}+\frac{L}{1-r}$ for $n \geq 0$.

Now assume that $x_{0} \neq x_{-1}$. From part (a) (with $i=0$ ) we have that for $n \geq 1$

$$
0 \leq 1+\sum_{k=1}^{n}(-1)^{k} \prod_{j=1}^{k}\left|H_{j}\right|<\frac{1}{1-r}
$$

If $-\left|H_{0}\right|\left(x_{0}-x_{-1}\right)<0$, then from Equation (19) we get that for $n \geq 0$

$$
x_{0} \geq x_{0}+\left(x_{0}-x_{-1}\right) \sum_{k=0}^{n}(-1)^{k+1} \prod_{j=0}^{k}\left|H_{j}\right| \geq x_{0}-\frac{\left|H_{0}\right|\left(x_{0}-x_{-1}\right)}{1-r}
$$

and so for $n \geq 0$

$$
\begin{aligned}
x_{0} & \geq x_{0}+\sum_{i=0}^{n} D_{i}\left(1+\sum_{k=1}^{n-i}(-1)^{k} \prod_{j=1+i}^{k+i}\left|H_{j}\right|\right) \\
& \geq x_{0}+\left(x_{0}-x_{-1}\right) \sum_{i=0}^{n}(-1)^{i+1} \prod_{j=0}^{i}\left|H_{j}\right|+\sum_{i=0}^{n} D_{i}\left(1+\sum_{k=1}^{n-i}(-1)^{k} \prod_{j=1+i}^{k+i}\left|H_{j}\right|\right) \\
& \geq x_{0}-\frac{\left|H_{0}\right|\left(x_{0}-x_{-1}\right)}{1-r}+\sum_{i=0}^{n} D_{i}\left(1+\sum_{k=1}^{n-i}(-1)^{k} \prod_{j=1+i}^{k+i}\left|H_{j}\right|\right) \\
& \geq x_{0}-\frac{\left|H_{0}\right|\left(x_{0}-x_{-1}\right)}{1-r}+\frac{L}{1-r} .
\end{aligned}
$$

Thus $x_{0} \geq x_{n+1} \geq x_{0}+\frac{L-\left|H_{0}\right|\left(x_{0}-x_{-1}\right)}{1-r}$ for $n \geq 0$.

If $\left|H_{0}\right|\left(x_{0}-x_{-1}\right)<0$, then from Equation (19) we get that for $n \geq 0$

$$
x_{0} \leq x_{0}+\left(x_{0}-x_{-1}\right)\left(\sum_{i=0}^{n}(-1)^{i+1} \prod_{j=0}^{i}\left|H_{j}\right|\right) \leq x_{0}-\frac{\left|H_{0}\right|\left(x_{0}-x_{-1}\right)}{1-r}
$$


and so

$$
\begin{aligned}
x_{0}+\frac{L}{1-r} & \leq x_{0}+\sum_{i=0}^{n} D_{i}\left(1+\sum_{k=1}^{n-i}(-1)^{k} \prod_{j=1+i}^{k+i}\left|H_{j}\right|\right) \\
& \leq x_{0}+\left(x_{0}-x_{-1}\right) \sum_{i=0}^{n}(-1)^{i+1} \prod_{j=0}^{i}\left|H_{j}\right|+\sum_{i=0}^{n} D_{i}\left(1+\sum_{k=1}^{n-i}(-1)^{k} \prod_{j=1+i}^{k+i}\left|H_{j}\right|\right) \\
& \leq x_{0}-\frac{\left|H_{0}\right|\left(x_{0}-x_{-1}\right)}{1-r}+\sum_{i=0}^{n} D_{i}\left(1+\sum_{k=1}^{n-i}(-1)^{k} \prod_{j=1+i}^{k+i}\left|H_{j}\right|\right) \\
& \leq x_{0}-\frac{\left|H_{0}\right|\left(x_{0}-x_{-1}\right)}{1-r} .
\end{aligned}
$$

Thus for $n \geq 0$

$$
x_{0}+\frac{L}{1-r} \leq x_{n+1} \leq x_{0}-\frac{\left|H_{0}\right|\left(x_{0}-x_{-1}\right)}{1-r}
$$

and so every solution is bounded.

(3) Suppose that $g_{1}=0$ for all $n \geq 0$. Then Equation (18) becomes $x_{n+1}=x_{n}+D_{n}, n=0,1, \ldots$, and so $x_{n+1}=x_{0}+\sum_{i=0}^{n} D_{i}, n=0,1, \ldots$, from which (a), (b) and (c) follow.

(d) Let $a \in \mathbb{R} \backslash\{0\}$ and assume that for $i=0,1, \ldots, D_{2 i}=a$ and $D_{2 i+1}=-a$. Equation (18) gives for $n=0,1, \ldots$,

$$
x_{2 n+1}=x_{0}+\sum_{i=0}^{2 n} D_{i}=x_{0}+a, \quad x_{2 n+2}=x_{0}+\sum_{i=0}^{2 n+1} D_{i}=x_{0} .
$$

(4) Suppose that $g_{1}=0$ for finite number of $n \geq 0$. By Lemma 3 part (b) with $F_{n}=-H_{n}$ we get that

$$
x_{n+1}=x_{0}+\left(x_{0}-x_{-1}\right) \sum_{i=0}^{n}(-1)^{i+1} \prod_{j=0}^{i} H_{j}+\sum_{i=0}^{n} D_{i}\left(1+\sum_{k=1}^{n-i}(-1)^{k} \prod_{j=1+i}^{k+i} H_{j}\right) .
$$

Let $N \geq 0$ be the first $n$ such that $g_{1}=0$ and let $M=0,1, \ldots$, be the last $n \geq 0$ such that $g_{1}=0$. Then $H_{N}=H_{M}=0$.

(a) Assume that $D_{n}=0$ for all $n \geq 0$. Then by Equation (20) for $n \geq N-1$

$$
x_{n+1}=x_{0}+\left(x_{0}-x_{-1}\right) \sum_{i=0}^{N-1}(-1)^{i+1} \prod_{j=0}^{i} H_{j} .
$$

(b) Assume that $D_{n} \neq 0$ for some $n \geq 0$. Then by Equation (20) for $n \geq M$

$$
\begin{aligned}
x_{n+1} & =x_{0}+\left(x_{0}-x_{-1}\right) \sum_{i=0}^{N-1}(-1)^{i+1} \prod_{j=0}^{i} H_{j}+\sum_{i=0}^{M-1} D_{i}\left(1+\sum_{k=1}^{M-i-1}(-1)^{k} \prod_{j=1+i}^{k+i} H_{j}\right) \\
& +\sum_{i=M}^{n} D_{i}\left(1+\sum_{k=1}^{n-i}(-1)^{k} \prod_{j=1+i}^{k+i} H_{j}\right) \\
& =\text { constant }+\sum_{i=M}^{n} D_{i}+\sum_{i=M}^{n} D_{i}\left(\sum_{k=1}^{n-i}(-1)^{k} \prod_{j=1+i}^{k+i} H_{j}\right) .
\end{aligned}
$$

(5) Suppose that $g_{1}=1$ for all $n \geq 0$. Then Equation (18) becomes $x_{n+1}=x_{n-1}+D_{n}, n=0,1, \ldots$ Thus for $n \geq 0$

$$
x_{2 n+1}=x_{-1}+\sum_{i=0}^{n} D_{2 i}, \quad x_{2 n+2}=x_{0}+\sum_{i=0}^{n} D_{2 i+1} .
$$

(a) If $D_{n}=0$ for all $n \geq 0$, then $x_{2 n+1}=x_{-1}$ and $x_{2 n+2}=x_{0}$ for $n=0,1, \ldots$

(b) If $\sum_{i=0}^{\infty} D_{2 i}= \pm \infty$, then $x_{2 n+1} \rightarrow \pm \infty$ as $n \rightarrow \infty$. If $\sum_{i=0}^{\infty} D_{2 i+1}= \pm \infty$, then $x_{2 n+2} \rightarrow \pm \infty$ as $n \rightarrow \infty$.

(c) If $\sum_{i=0}^{\infty} D_{2 i}$ converges and $\sum_{i=0}^{\infty} D_{2 i+1}$ converges, then $x_{2 n+1}$ and $x_{2 n+2}$ converge as $n \rightarrow \infty$.

(6) Suppose that $1<r \leq g_{1}$ for all $n \geq 0$. Then $1<r \leq H_{n}$ for all $n \geq 0$. From Lemma 3 part (b) with $F_{n}=-H_{n}$ we get that for $n \geq 0$ 


$$
\begin{aligned}
x_{2 n+2} & =x_{0}+\left(x_{0}-x_{-1}\right) \sum_{i=0}^{2 n+1}(-1)^{i+1} \prod_{j=0}^{i} H_{j}+\sum_{i=0}^{2 n+1} D_{i}\left(1+\sum_{k=1}^{2 n+1-i}(-1)^{k} \prod_{j=1+i}^{k+i} H_{j}\right) \\
& =x_{0}+\left(x_{0}-x_{-1}\right) \sum_{i=0}^{n}\left(H_{2 i+1}-1\right) \prod_{j=0}^{2 i} H_{j}-\sum_{i=0}^{n} D_{2 i}\left(\sum_{k=0}^{n-i}\left(H_{2 k+2 i+1}-1\right) \prod_{j=1+2 i}^{2 k+2 i} H_{j}\right) \\
& +\sum_{i=0}^{n} D_{2 i+1}\left(1+\sum_{k=1}^{n-i}\left(H_{2 k+2 i+1}-1\right) \prod_{j=2+2 i}^{2 k+2 i} H_{j}\right) .
\end{aligned}
$$

Observe that for $n \geq 0,(r-1) r^{2 n}<\left(H_{2 n+1}-1\right) \prod_{j=0}^{2 n} H_{j}$ and $\lim _{n \rightarrow \infty} r^{2 n-N}=\infty$ for any fixed $N$. Let $N$ be the first $n \geq 0$ such that $D_{n} \neq 0$. Suppose that $N$ is even. Then for $n \geq N / 2$ we have that

$$
\begin{aligned}
x_{2 n+2} & =x_{0}+\left(x_{0}-x_{-1}\right) \sum_{i=0}^{n-1}\left(H_{2 i+1}-1\right) \prod_{j=0}^{2 i} H_{j}+\left(x_{0}-x_{-1}\right)\left(H_{2 n+1}-1\right) \prod_{j=0}^{2 n} H_{j} \\
& -D_{N} \sum_{k=0}^{n-N / 2}\left(H_{2 k+N+1}-1\right) \prod_{j=1}^{2 k} H_{j+N}-\sum_{i=\frac{N+2}{2}}^{n} D_{2 i} \sum_{k=0}^{n-i}\left(H_{2 k+2 i+1}-1\right) \prod_{j=1+2 i}^{2 k+2 i} H_{j} \\
& +\sum_{i=N / 2}^{n} D_{2 i+1}\left(1+\sum_{k=1}^{n-i}\left(H_{2 k+2 i+1}-1\right) \prod_{j=2+2 i}^{2 k+2 i} H_{j}\right) .
\end{aligned}
$$

For $n>N / 2$

$$
\begin{aligned}
(r-1) r^{2 n-N} & <(r-1)+(r-1) r^{2}+\ldots+(r-1) r^{2 n-N} \\
& \leq\left(H_{N+1}-1\right)+\left(H_{N+3}-1\right) \prod_{j=1}^{2} H_{j+N}+\ldots+\left(H_{2 n+1}-1\right) \prod_{j=1}^{2 n-N} H_{j+N} \\
& =\sum_{k=0}^{n-N / 2}\left(H_{2 k+N+1}-1\right) \prod_{j=1}^{2 k} H_{j+N} .
\end{aligned}
$$

(a) Assume that $x_{0} \geq x_{-1}, D_{2 n} \leq 0, D_{2 n+1} \geq 0, n=0,1, \ldots$. Then for $n \geq N / 2$

$$
\begin{aligned}
x_{2 n+2} & =x_{0}+\left(x_{0}-x_{-1}\right)\left(H_{2 n+1}-1\right) \prod_{j=0}^{2 n} H_{j}-D_{N} \sum_{k=0}^{n-N / 2}\left(H_{2 k+N+1}-1\right) \prod_{j=0}^{2 k} H_{j+N}+R_{n} \\
& >x_{0}+\left(x_{0}-x_{-1}\right)(r-1) r^{2 n}-D_{N}(r-1) r^{2 n-N} .
\end{aligned}
$$

Thus $x_{2 n+2} \rightarrow \infty$ as $n \rightarrow \infty$.

(b) Assume that $x_{0} \leq x_{-1}, D_{2 n} \geq 0, D_{2 n+1} \leq 0, n=0,1, \ldots$ Then for $n>N / 2$

$$
\begin{aligned}
x_{2 n+2} & =x_{0}+\left(x_{0}-x_{-1}\right)\left(H_{2 n+1}-1\right) \prod_{j=0}^{2 n} H_{j}-D_{N} \sum_{k=0}^{n-N / 2}\left(H_{2 k+N+1}-1\right) \prod_{j=0}^{2 k} H_{j+N}+S_{n} \\
& <x_{0}+\left(x_{0}-x_{-1}\right)(r-1) r^{2 n}-D_{N}(r-1) r^{2 n-N} .
\end{aligned}
$$

Thus $x_{2 n+2} \rightarrow-\infty$ as $n \rightarrow \infty$.

Now suppose that $N$ is odd. Then for $n \geq N$ we have that

$$
\begin{aligned}
x_{2 n+2} & =x_{0}+\left(x_{0}-x_{-1}\right) \sum_{i=0}^{n-1}\left(H_{2 i+1}-1\right) \prod_{j=0}^{2 i} H_{j}+\left(x_{0}-x_{-1}\right)\left(H_{2 n+1}-1\right) \prod_{j=0}^{2 n} H_{j} \\
& +D_{N}\left(1+\sum_{k=1}^{n-\frac{N-1}{2}}\left(H_{2 k+N}-1\right) \prod_{j=1}^{2 k-1} H_{j+N}\right) \\
& +\sum_{i=\frac{N+1}{2}}^{n} D_{2 i+1}\left(1+\sum_{k=1}^{n-i}\left(H_{2 k+2 i+1}-1\right) \prod_{j=2+2 i}^{2 k+2 i} H_{j}\right) \\
& -\sum_{i=\frac{N+1}{2}}^{n} D_{2 i}\left(\sum_{k=0}^{n-i}\left(H_{2 k+2 i+1}-1\right) \prod_{j=1+2 i}^{2 k+2 i} H_{j}\right) .
\end{aligned}
$$

For $n \geq N$

$$
\begin{aligned}
(r-1) r^{2 n-N} & <1+(r-1)+(r-1) r^{2}+\ldots+(r-1) r^{2 n-N} \\
& \leq 1+\left(H_{N+2}-1\right) \prod_{j=1}^{1} H_{j+N}+\ldots+\left(H_{2 n+1}-1\right) \prod_{j=1}^{2 n-N} H_{j+N} \\
& =1+\sum_{k=1}^{n-\frac{N-1}{2}}\left(H_{2 k+N}-1\right) \prod_{j=1}^{2 k-1} H_{j+N} .
\end{aligned}
$$

(a) Assume that $x_{0} \geq x_{-1}, D_{2 n} \leq 0, D_{2 n+1} \geq 0, n=0,1, \ldots$. Then for $n \geq N$

$$
\begin{aligned}
x_{2 n+2} & =x_{0}+\left(x_{0}-x_{-1}\right)\left(H_{2 n+1}-1\right) \prod_{j=0}^{2 n} H_{j}+D_{N}\left(1+\sum_{k=1}^{n-\frac{N-1}{2}}\left(H_{2 k+N}-1\right) \prod_{j=0}^{2 k-1} H_{j+N}\right)+R_{n} \\
& >x_{0}+\left(x_{0}-x_{-1}\right)(r-1) r^{2 n}+D_{N}(r-1) r^{2 n-N} .
\end{aligned}
$$

Thus $x_{2 n+2} \rightarrow \infty$ as $n \rightarrow \infty$.

(b) Assume that $x_{0} \leq x_{-1}, D_{2 n} \geq 0, D_{2 n+1} \leq 0, n=0,1, \ldots$. Then for $n \geq N$ 


$$
\begin{aligned}
x_{2 n+2} & =x_{0}+\left(x_{0}-x_{-1}\right)\left(H_{2 n+1}-1\right) \prod_{j=0}^{2 n} H_{j}+D_{N}\left(1+\sum_{k=1}^{n-\frac{N-1}{2}}\left(H_{2 k+N}-1\right) \prod_{j=1}^{2 k-1} H_{j+N}\right)+S_{n} \\
& <x_{0}+\left(x_{0}-x_{-1}\right)(r-1) \prod_{j=0}^{2 n} H_{j}+D_{N}(r-1) r^{2 n-N}
\end{aligned}
$$

Thus $x_{2 n+2} \rightarrow-\infty$ as $n \rightarrow \infty$.

Example 4. The difference equation

$$
x_{n+1}=\frac{x_{n} e^{-x_{n}}+(a+1) x_{n}-x_{n-1}}{a+e^{-x_{n}}}, \quad n=0,1, \ldots,
$$

where $a \geq 0$, when rewritten as

$$
x_{n+1}=\frac{e^{-x_{n}}+(a+1)}{a+e^{-x_{n}}} x_{n}+\frac{-1}{a+e^{-x_{n}}} x_{n-1}=g_{0} x_{n}+g_{1} x_{n-1}, \quad n=0,1, \ldots,
$$

satisfies all conditions of Theorem 1 part (2a) when $a>1$, that is $g_{0}+g_{1}=1$ and $\left|g_{1}\right|=\frac{1}{a+e^{-x_{n}}} \leq \frac{1}{a}$ and so every solution of Equation (21), when a $>1$ converges to a limit point.

In addition, Equation (21) implies

$$
x_{n+1}-x_{n}=\frac{x_{n}-x_{n-1}}{a+e^{-x_{n}}}, \quad n=0,1, \ldots,
$$

which shows that when $x_{0}>x_{-1}$ (resp. $\left.x_{0}<x_{-1}\right)$ the solution $\left\{x_{n}\right\}$ is increasing (resp. decreasing) and so it has to have a finite or infinite limit.

Suppose that $0 \leq a \leq 1$. Assume that $x_{0}<x_{-1}$ and $x_{0}<0$. Then $e^{-x_{0}}>1$ and so there exists $c \in(0, \infty)$ such that $e^{-x_{n}} \geq e^{-x_{0}} \geq c>1$ for $n \geq 0$. Thus for $n \geq 0$

$$
0>\frac{-1}{a+e^{-x_{n}}} \geq \frac{-1}{a+c}>-1
$$

Therefore by Theorem 1 part (2a) $\lim _{n \rightarrow \infty} x_{n}=L \in(-\infty, \infty)$.

Now suppose that $a \in[0,1)$. Assume that $x_{0}>x_{-1}$ such that $x_{0} \geq-\ln (1-a)$. Then $e^{-x_{n}} \leq e^{-x_{0}} \leq$ $1-a$ and so $\frac{-1}{a+e^{-x_{n}}} \leq-1$ for $n \geq 0$. Thus by Theorem 1 part (1a) $\lim _{n \rightarrow \infty} x_{n}=\infty$.

In some very special cases of the terms $D_{n}$ in Equation (18) one can obtain results not included in Theorem 1. We illustrate this with the following result.

Theorem 2. Consider the difference equation

$$
x_{n+1}=A_{n} x_{n}+B_{n} x_{n-1}+D_{n}, \quad n=0,1, \ldots
$$

where $A_{n}, B_{n} \in \mathbb{R} \backslash\{0\}$ such that $A_{n}+B_{n}=1$ for all $n \geq 0$. Let $r \in(0, \infty)$. Then every solution of Equation (24) is unbounded provided one of the following holds:

(1) Assume that $-1<-r \leq B_{n}<0$ and either $\sum_{i=0}^{\infty} D_{i}=\infty$, where $D_{n} \geq 0$ or $\sum_{i=0}^{\infty} D_{i}=-\infty$, where $D_{n} \leq 0$ for $n \geq 0$;

(2) Assume that $0<B_{n} \leq r<1$ for $n \geq 0$.

(a) Suppose that for all $n \geq 0$ either

$D_{2 n+1} \geq B_{2 n+1} D_{2 n}, D_{n} \geq 0$ and $\sum_{i=0}^{\infty} D_{2 i}=\infty$ or $D_{2 n+1} \leq B_{2 n+1} D_{2 n}, D_{n} \leq 0$ and $\sum_{i=0}^{\infty} D_{2 i}=-\infty$;

(b) Suppose that for all $n \geq 0$ either

$D_{2 n+1} \leq B_{2 n+1} D_{2 n}, D_{n} \geq 0$ and $\sum_{i=0}^{\infty} D_{2 i+1}=\infty$ or $D_{2 n+1} \geq B_{2 n+1} D_{2 n}, D_{n} \leq 0$ and $\sum_{i=0}^{\infty} D_{2 i+1}=-\infty$. 
Proof. First we rewrite Equation (24) in the form

$$
x_{n+1}=x_{n}-B_{n}\left(x_{n}-x_{n-1}\right)+D_{n}, \quad n=0,1, \ldots
$$

Let $R_{n}$ (resp. $S_{n}$ ) be the sum of positive (resp. negative) terms.

(1) Assume that $-1<-r \leq B_{n}<0$ for $n \geq 0$. Setting $F_{n}=-B_{n}$ Lemma 3 part (b) implies for $n \geq 0$

$$
x_{n+1}=x_{0}+\left(x_{0}-x_{-1}\right) \sum_{i=0}^{n} \prod_{j=0}^{i} F_{j}+\sum_{i=0}^{n} D_{i}\left(\sum_{k=0}^{n-i} \prod_{j=1+i}^{k+i} F_{j}\right)+\sum_{i=0}^{n} D_{i} .
$$

Since $0<\prod_{j=0}^{i} F_{j} \leq r^{i}$ for $i=0,1, \ldots$, then the series $\sum_{i=0}^{n} \prod_{j=0}^{i} F_{j}$ converges as $n \rightarrow \infty$.

If for $n \geq 0, D_{n} \geq 0$ and $\sum_{i=0}^{\infty} D_{i}=\infty$, then

$$
x_{n+1}=x_{0}+\left(x_{0}-x_{-1}\right) \sum_{i=0}^{n} \prod_{j=0}^{i} F_{j}+\sum_{i=0}^{n} D_{i}+R_{n} .
$$

Thus $x_{n} \rightarrow \infty$ as $n \rightarrow \infty$.

If for $n \geq 0, D_{n} \leq 0$ and $\sum_{i=0}^{\infty} D_{i}=-\infty$, then

$$
x_{n+1}=x_{0}+\left(x_{0}-x_{-1}\right) \sum_{i=0}^{n} \prod_{j=0}^{i} F_{j}+\sum_{i=0}^{n} D_{i}+S_{n} .
$$

Thus $x_{n} \rightarrow-\infty$ as $n \rightarrow \infty$.

(2) Assume that $0<B_{n} \leq r<1$ for $n \geq 0$.

(a) By Lemma 3 part (b) with $F_{n}=B_{n}$ we get that for $n \geq 0$

$$
\begin{aligned}
x_{2 n+1} & =x_{0}+\left(x_{0}-x_{-1}\right) \sum_{i=0}^{2 n}(-1)^{i+1} \prod_{j=0}^{i} B_{j}+\sum_{i=0}^{2 n} D_{i}\left(1+\sum_{k=1}^{n-i}(-1)^{k} \prod_{j=1+i}^{k+i} B_{j}\right) \\
& =x_{0}+\left(x_{0}-x_{-1}\right) \sum_{i=0}^{2 n}(-1)^{i+1} \prod_{j=0}^{i} B_{j}+\sum_{i=0}^{n} D_{2 i}\left(1-\sum_{k=1}^{n-i}\left(1-B_{2 k+2 i}\right) \prod_{j=1+2 i}^{2 k+2 i-1} B_{j}\right) \\
& +\sum_{i=0}^{n} D_{2 i+1}\left(\sum_{k=1}^{n-i}\left(1-B_{2 k+2 i}\right) \prod_{j=2+2 i}^{2 k+2 i-1} B_{j}\right) \\
& =x_{0}+\left(x_{0}-x_{-1}\right) \sum_{i=0}^{2 n}(-1)^{i+1} \prod_{j=0}^{i} B_{j}+\sum_{i=0}^{n} D_{2 i} \\
& +\sum_{i=0}^{n}\left(D_{2 i+1}-B_{2 i+1} D_{2 i}\right)\left(\sum_{k=1}^{n-i}\left(1-B_{2 k+2 i}\right) \prod_{j=2+2 i}^{2 k+2 i-1} B_{j}\right) .
\end{aligned}
$$

Observe that the series $\sum_{i=0}^{\infty}(-1)^{i+1} \prod_{j=0}^{i} B_{j}$ converges by the alternating series test. If $D_{2 n+1} \geq B_{2 n+1} D_{2 n}, D_{n} \geq 0$ and $\sum_{i=0}^{\infty} D_{2 i}=\infty$, then for $n \geq 0$

$$
x_{2 n+1}=x_{0}+\left(x_{0}-x_{-1}\right) \sum_{i=0}^{2 n}(-1)^{i+1} \prod_{j=0}^{i} B_{j}+\sum_{i=0}^{n} D_{2 i}+R_{n} .
$$

Thus $x_{2 n+1} \rightarrow \infty$ as $n \rightarrow \infty$.

If $D_{2 n+1} \leq B_{2 n+1} D_{2 n}, D_{n} \leq 0$ and $\sum_{i=0}^{\infty} D_{2 i}=-\infty$, then for $n \geq 0$

$$
x_{2 n+1}=x_{0}+\left(x_{0}-x_{-1}\right) \sum_{i=0}^{2 n}(-1)^{i+1} \prod_{j=0}^{i} B_{j}+\sum_{i=0}^{n} D_{2 i}+S_{n} .
$$

Thus $x_{2 n+1} \rightarrow-\infty$ as $n \rightarrow \infty$. 
(b) By Lemma 3 part (b) with $F_{n}=B_{n}$ we get that for $n \geq 0$

$$
\begin{aligned}
x_{2 n+2} & =x_{0}+\left(x_{0}-x_{-1}\right) \sum_{i=0}^{2 n+1}(-1)^{i+1} \prod_{j=0}^{i} B_{j}+\sum_{i=0}^{2 n+1} D_{i}\left(1+\sum_{k=1}^{2 n+1-i}(-1)^{k} \prod_{j=1+i}^{k+i} B_{j}\right) \\
& =x_{0}+\left(x_{0}-x_{-1}\right) \sum_{i=0}^{2 n+1}(-1)^{i+1} \prod_{j=0}^{i} B_{j}+\sum_{i=0}^{n} D_{2 i+1}\left(1-\sum_{k=1}^{n-i}\left(1-B_{2 k+2 i+1}\right) \prod_{j=2+2 i}^{2 k+2 i} B_{j}\right) \\
& +\sum_{i=0}^{n} D_{2 i}\left(1-B_{2 i+1}+\sum_{k=1}^{n-i}\left(1-B_{2 k+2 i+1}\right) \prod_{j=1+2 i}^{2 k+2 i} B_{j}\right) \\
& =x_{0}+\left(x_{0}-x_{-1}\right) \sum_{i=0}^{2 n+1}(-1)^{i+1} \prod_{j=0}^{i} B_{j}+\sum_{i=0}^{n} D_{2 i}\left(1-B_{2 i+1}\right)+\sum_{i=0}^{n} D_{2 i+1} \\
& +\sum_{i=0}^{n}\left(B_{2 i+1} D_{2 i}-D_{2 i+1}\right)\left(\sum_{k=1}^{n-i}\left(1-B_{2 k+2 i+1}\right) \prod_{j=2+2 i}^{2 k+2 i} B_{j}\right) .
\end{aligned}
$$

If $D_{2 n+1} \leq B_{2 n+1} D_{2 n}, D_{n} \geq 0$ and $\sum_{i=0}^{\infty} D_{2 i+1}=\infty$, then for $n \geq 0$

$$
x_{2 n+2}=x_{0}+\left(x_{0}-x_{-1}\right) \sum_{i=0}^{2 n+1}(-1)^{i+1} \prod_{j=0}^{i} B_{j}+\sum_{i=0}^{n} D_{2 i+1}+R_{n} .
$$

Thus $x_{2 n+2} \rightarrow \infty$ as $n \rightarrow \infty$.

If $D_{2 n+1} \geq B_{2 n+1} D_{2 n}, D_{n} \leq 0$ and $\sum_{i=0}^{\infty} D_{2 i+1}=-\infty$, then for $n \geq 0$

$$
x_{2 n+2}=x_{0}+\left(x_{0}-x_{-1}\right) \sum_{i=0}^{2 n+1}(-1)^{i+1} \prod_{j=0}^{i} B_{j}+\sum_{i=0}^{n} D_{2 i+1}+S_{n} .
$$

Thus $x_{2 n+2} \rightarrow-\infty$ as $n \rightarrow \infty$.

Remark 3. In Theorem 1 cases (2) and (6) the existence of $r$ is a sufficient condition but not a necessary condition. To show this for case (2) consider Equation (3) where $n=2,3, \ldots$. Since $g_{1}=\frac{n-1}{n} \rightarrow 1$ as $n \rightarrow \infty$, there is no $r$ such that $g_{1} \leq r<1$. Rewriting Equation (3) as

$$
x_{n+1}=x_{n}-\frac{n-1}{n}\left(x_{n}-x_{n-1}\right), \quad n=2,3, \ldots,
$$

we find its solution as

$$
x_{n+1}=x_{2}-\left(x_{2}-x_{1}\right) \sum_{i=2}^{n}(-1)^{i} \frac{1}{i}, \quad n=2,3, \ldots,
$$

which converges to a constant.

Now for the case (5), consider the difference equation

$$
x_{n+1}=-\frac{1}{n} x_{n}+\frac{n+1}{n} x_{n-1}, \quad n=1,2, \ldots,
$$

Clearly $g_{0}+g_{1}=1, g_{1} \rightarrow 1$ as $n \rightarrow \infty$ and there is no $r$ such that $g_{1} \geq r>1$.

Rewriting Equation (25) as

$$
x_{n+1}=x_{n}-\frac{n+1}{n}\left(x_{n}-x_{n-1}\right), \quad n=1,2, \ldots,
$$

we find its solution as

$$
x_{n+1}=x_{1}+\left(x_{1}-x_{0}\right) \sum_{i=1}^{n}(-1)^{i}(i+1),
$$

which is unbounded.

Example 5. Consider the following equation

$$
x_{n+1}=\left(\frac{1}{2}+a_{n}\right) x_{n}+\left(\frac{1}{2}-a_{n}\right) x_{n-1}, \quad n=0,1, \ldots,
$$


where $a_{n}$ is a real sequence, which depends on $n$ and on one or more state variables $x_{n}$. An immediate application of Theorem 1 with $D_{n}=0, n=0,1, \ldots$, leads to the following result for Equation (26). Here $r$ is a positive constant.

Corollary 1. The global behavior of Equation (26) is as follows:

(a) If $\frac{3}{2} \leq a_{n}$ for $n \geq 0$, then every non-constant solution of Equation (26) is unbounded.

(b) If $0<\left|\frac{1}{2}-a_{n}\right| \leq r<1$ for $n \geq 0$, then every non-constant solution of Equation (26) converges to a limit.

(c) If $a_{n}=\frac{1}{2}$ for all $n \geq 0$, then $x_{n}=x_{0}$.

(d) If $a_{n}=\frac{1}{2}$ for a finite number of $n \geq 0$, then every solution of Equation (26) is eventually equal to a constant.

(e) If $a_{n}=-\frac{1}{2}$ for $n \geq 0$, then every non-constant solution of Equation (26) is a period-two solution.

(f) If $a_{n} \leq \frac{1}{2}-r<-\frac{1}{2}, r>1$ for $n \geq 0$, then every non-constant solution of Equation (26) is unbounded.

In the case when Equation (1) has periodic coefficients it can be reduced to an associated system of second order equations with constant coefficients which can be solved. We illustrate this case by the Example 6.

Example 6. Consider the following second order equation

$$
x_{n+1}=A_{n} x_{n}+B_{n} x_{n-1}, \quad n=0,1, \ldots,
$$

where $B_{n} \in \mathbb{R} \backslash\{0\}$ and $A_{n}+B_{n}=1$ for all $n \geq 0$. Assume that $A_{n}$ and $B_{n}$ are period-two sequences, that is

$$
A_{n}=A_{i}, \quad B_{n}=B_{i}, \quad n=2 j+i, i=0,1 ; j=0,1, \ldots
$$

A straight-forward simplification leads to the following autonomous system for even and odd indexed terms:

$$
\begin{aligned}
& x_{2 n+3}=\left(1+B_{0} B_{1}\right) x_{2 n+1}-B_{0} B_{1} x_{2 n-1} \\
& x_{2 n+4}=\left(1+B_{0} B_{1}\right) x_{2 n+2}-B_{0} B_{1} x_{2 n}, \quad n=0,1, \ldots
\end{aligned}
$$

Let $y_{n}^{\alpha}=x_{2 n+1+\alpha}, \alpha=0,1, n=0,1, \ldots$. Then Equation (27) is equivalent with the autonomous equation

$$
y_{n+1}^{\alpha}=\left(1+B_{0} B_{1}\right) y_{n}^{\alpha}-B_{0} B_{1} y_{n-1}^{\alpha}, \quad \alpha=0,1, n=0,1, \ldots .
$$

In view of Lemma 1 and Theorem 1, we have the following result.

Proposition 1. Consider Equation (27) with period-two coefficients where $B_{n} \in \mathbb{R} \backslash\{0\}$ and $A_{n}+B_{n}=1$ for all $n \geq 0$. Then

(1) If $\left|B_{0} B_{1}\right|<1$, then every nonconstant solution $\left\{x_{n}\right\}$ of Equation (27) converges to the limit

$$
\lim _{n \rightarrow \infty} x_{n}=\frac{\left(1-B_{0}\right) x_{0}+B_{0}\left(1-B_{1}\right) x_{-1}}{1-B_{0} B_{1}} ;
$$

(2) If $B_{0} B_{1}=-1$, then every nonconstant solution $\left\{x_{n}\right\}$ of Equation (27) is of period 4;

(3) If either $B_{0} B_{1} \geq 1$ or $B_{0} B_{1}<-1$, then every nonconstant solution $\left\{x_{n}\right\}$ of Equation (27) is unbounded.

Proof. By Theorem 1 with $D_{n}=0, n=0,1, \ldots$, we have

(1) If $\left|B_{0} B_{1}\right|<1$, then every nonconstant solution $\left\{y_{n}^{\alpha}\right\}$ of Equation (28) converges to the limit $L_{\alpha}$, that is

$$
\lim _{n \rightarrow \infty} y_{n}^{\alpha}=L_{\alpha}, \quad \alpha=0,1
$$


(2) If $B_{0} B_{1}=-1$, then every nonconstant solution $\left\{y_{n}^{\alpha}\right\}$ of Equation (28) is of period 2;

(3) If either $B_{0} B_{1} \geq 1$ or $B_{0} B_{1}<-1$, then every nonconstant solution $\left\{y_{n}^{\alpha}\right\}$ of Equation (28) is unbounded.

Now the results follow from (1)-(3). In particular, by Lemma 1 Equation (28) has the invariant $I_{n}^{\alpha}=y_{n}^{\alpha}-B_{0} B_{1} y_{n-1}^{\alpha}$, where $\alpha=0,1$ and $n=0,1, \ldots$ If $\left|B_{0} B_{1}\right|<1$, then every nonconstant solution $\left\{y_{n}^{\alpha}\right\}$ of Equation (28) converges to the limit $L_{\alpha}$, which implies

$$
\lim _{n \rightarrow \infty} I_{n}^{\alpha}=L_{\alpha}\left(1-B_{0} B_{1}\right)=I_{0}^{\alpha}=y_{0}^{\alpha}-B_{0} B_{1} y_{-1}^{\alpha} .
$$

Now by using Equation (17), Lemma 1 and Theorem 1 we can obtain explicitly the behavior of the solutions of Equation (1) when $g_{0}$ and $g_{1}$ are periodic and condition (2) is satisfied.

Corollary 2. Let $p \in\{1,2, \ldots\}$ and let $B_{n} \in R \backslash\{0\}, n \geq 0$. Consider Equation (27) where $A_{n}+B_{n}=1$ for all $n \geq 0$ and that

$$
A_{n}=A_{k}, \quad B_{n}=B_{k}, \quad k=0,1, \ldots, p-1, n=p j+k ; j=0,1, \ldots
$$

(a) If either $(-1)^{p-1} \prod_{i=0}^{p-1} B_{i} \leq-1$ or $(-1)^{p-1} \prod_{i=0}^{p-1} B_{i}>1$, then every non-constant solution $\left\{x_{n}\right\}$ of Equation (27) is unbounded.

(b) If $(-1)^{p-1} \prod_{i=0}^{p-1} B_{i}=1$, then every non-constant solution $\left\{x_{n}\right\}$ of Equation (27) is periodic of period $2 p$. In particular, the periodic solution is $\left\{x_{-1}, x_{\gamma}\right\}$ where

$$
x_{\gamma}=x_{0}+\left(x_{0}-x_{-1}\right) \sum_{i=0}^{\gamma-1}(-1)^{i+1} \prod_{j=0}^{i} B_{j}
$$

and $\gamma=0,1, \ldots, 2 p-2$.

(c) If $\left|\prod_{i=0}^{p-1} B_{i}\right|<1$, then every non-constant solution $\left\{x_{n}\right\}$ of Equation (27) converges to a limit, that is

$$
\lim _{n \rightarrow \infty} x_{n}=\frac{\left(1+\sum_{j=0}^{p-2}(-1)^{j+1} \prod_{i=0}^{j} B_{i}\right) x_{0}+\left(\sum_{j=0}^{p-1}(-1)^{j} \prod_{i=0}^{j} B_{i}\right) x_{-1}}{1+(-1)^{p-1} \prod_{i=0}^{p-1} B_{i}} .
$$

Proof. Equation (27) implies that for $\beta=0,1, \ldots$

$$
x_{p n+\beta+1}=\left(1-B_{\beta}\right) x_{p n+\beta}+B_{\beta} x_{p n+\beta-1}, \quad n=0,1, \ldots
$$

and so

$$
\left(1-B_{\beta}\right) x_{p n+\beta}=x_{p n+\beta+1}-B_{\beta} x_{p n+\beta-1} .
$$

Then for $\beta=0,1, \ldots ; n \geq 0$

$$
\left(1+\sum_{j=0}^{\beta}(-1)^{j+1} \prod_{i=0}^{j} B_{i}\right) x_{p n+\beta}=\left(1+\sum_{j=0}^{\beta-1}(-1)^{j+1} \prod_{i=0}^{j} B_{i}\right) x_{p n+\beta+1}+(-1)^{\beta+1} \prod_{i=0}^{\beta} B_{i} x_{p n-1} .
$$

Indeed for $\beta=0,1, \ldots$, we have that for $n \geq 0$

$$
\begin{gathered}
\left(1-B_{\beta+1}\right)\left(1+\sum_{j=0}^{\beta}(-1)^{j+1} \prod_{i=0}^{j} B_{i}\right) x_{p n+\beta+1}=\left(1+\sum_{j=0}^{\beta}(-1)^{j+1} \prod_{i=0}^{j} B_{i}\right) x_{p n+\beta+2} \\
-\left(1+\sum_{j=0}^{\beta}(-1)^{j+1} \prod_{i=0}^{j} B_{i}\right) B_{p+1} x_{p n+\beta} .
\end{gathered}
$$


Multiplying Equation (30) by $-B_{\beta+1}$ and substituting into Equation (31) we obtain

$$
\left(1+\sum_{j=0}^{\beta+1}(-1)^{j+1} \prod_{i=0}^{j} B_{i}\right) x_{p n+\beta+1}=\left(1+\sum_{j=0}^{\beta}(-1)^{j+1} \prod_{i=0}^{j} B_{i}\right) x_{p n+\beta+2}+(-1)^{\beta+2} \prod_{i=0}^{\beta+1} B_{i} x_{p n-1} .
$$

Now from Equation (29) when $\beta=p-1$ we have that for $n \geq 0$

$$
x_{p n+p}=\left(1-B_{p-1}\right) x_{p n+p-1}+B_{p-1} x_{p n+p-2} .
$$

By using Equation (29) with $\beta=p$ and Equation (32) we obtain

$$
x_{p n+p+1}=\left(1-B_{p-1}+B_{p-1} B_{p}\right) x_{p n+p-1}+B_{p-1}\left(1-B_{p}\right) x_{p n+p-2} .
$$

Now continuing this process we get that for $\delta=0,1, \ldots$

$$
x_{p n+p+\delta}=\left(1+\sum_{j=0}^{\delta}(-1)^{j+1} \prod_{i=0}^{j} B_{i+p-1}\right) x_{p n+p-1}+B_{p-1}\left(1+\sum_{j=0}^{\delta-1}(-1)^{j+1} \prod_{i=0}^{j} B_{i+p}\right) x_{p n+p-2} .
$$

Observe that since $B_{i}=B_{i+p}$ for $i=0,1, \ldots$, then

$$
\begin{aligned}
\sum_{j=0}^{p-1}(-1)^{j+1} \prod_{i=0}^{j} B_{i+p-1} & =\sum_{j=0}^{p-2}(-1)^{j+1} \prod_{i=0}^{j} B_{i+p-1}+(-1)^{p} \prod_{i=0}^{p-1} B_{i+p-1} \\
& =-B_{p-1}\left(1+\sum_{j=0}^{p-3}(-1)^{j+1} \prod_{i=0}^{j} B_{i}\right)+(-1)^{p} \prod_{i=0}^{p-1} B_{i} .
\end{aligned}
$$

Now multiplying Equation (30) where $\beta=p-2$ with $B_{p-1}$ and substituting into Equation (33) where $\delta=p-1$ we obtain

$$
x_{p n+2 p-1}=\left(1+(-1)^{p} \prod_{i=0}^{p-1} B_{i}\right) x_{p n+p-1}+(-1)^{p-1} \prod_{i=0}^{p-1} B_{i} x_{p n-1} .
$$

Similarly, we can show that for $\alpha=1,2, \ldots, p-1$

$$
x_{p n+2 p-1+\alpha}=\left(1+(-1)^{p} \prod_{i=0}^{p-1} B_{i}\right) x_{p n+p-1+\alpha}+(-1)^{p-1} \prod_{i=0}^{p-1} B_{i} x_{p n-1+\alpha} .
$$

For $\alpha=0, \ldots, p-1$ let $y_{n}^{\alpha}=x_{p n+p-1+\alpha}, n=0,1, \ldots$. Then for $\alpha=0, \ldots, p-1$, $y_{n+1}^{\alpha}=x_{p n+2 p-1+\alpha}$ and $y_{n-1}^{\alpha}=x_{p n-1+\alpha}$. Thus for $\alpha=0, \ldots, p-1$ Equation (34) becomes

$$
y_{n+1}^{\alpha}=\left(1+(-1)^{p} \prod_{i=0}^{p-1} B_{i}\right) y_{n}^{\alpha}+(-1)^{p-1} \prod_{i=0}^{p-1} B_{i} y_{n-1}^{\alpha} \text {. }
$$

(a) Suppose that either $(-1)^{p-1} \prod_{i=0}^{p-1} B_{i} \leq-1$ or $(-1)^{p-1} \prod_{i=0}^{p-1} B_{i}>1$. By Theorem 1 parts (1) and (6), where $g_{1}=(-1)^{p-1} \prod_{i=0}^{p-1} B_{i}$, and $D_{n}=0, n=0,1, \ldots$, every non-constant solution $\left\{y_{n}^{\alpha}\right\}, \alpha=0,1, \ldots, p-1$ of Equation (35) is unbounded. Thus every non-constant subsequence $\left\{x_{p n+p-1+\alpha}\right\}$ of Equation (27) is unbounded.

(b) Suppose that $(-1)^{p-1} \prod_{i=0}^{p-1} B_{i}=1$. Then for $\alpha=0,1, \ldots, p-1$ Equation (35) becomes $y_{n+1}^{\alpha}=y_{n-1}^{\alpha}, n=0,1, \ldots$. Thus for $\alpha=0,1, \ldots, p-1$ the solution $\left\{y_{n}^{\alpha}\right\}$ is a period-two sequence. Since there are $p$ subsequences, then every non-constant solution $\left\{x_{n}\right\}$ of Equation (27) is periodic of period $2 p$.

(c) Suppose that $\left|\prod_{i=0}^{p-1} B_{i}\right|<1$. Then by Theorem 1 part (2), where $g_{1}=(-1)^{p-1} \prod_{i=0}^{p-1} B_{i}$ and $D_{n}=0, n=0,1, \ldots$, every non-constant solution $\left\{y_{n}^{\alpha}\right\}, \alpha=0,1, \ldots, p-1$ of Equation (35) converges to a limit point. Let $L^{\alpha}=\lim _{n \rightarrow \infty} y_{n}^{\alpha}$ for $\alpha=0, \ldots, p-1$. Then by Lemma 1 


$$
L^{\alpha}\left(1+(-1)^{p-1} \prod_{i=0}^{p-1} B_{i}\right)=y_{0}^{\alpha}+\left((-1)^{p-1} \prod_{i=0}^{p-1} B_{i}\right) y_{-1}^{\alpha} .
$$

Thus for $\alpha=0, \ldots, p-1$

$$
\lim _{n \rightarrow \infty} y_{n}^{\alpha}=\frac{y_{0}^{\alpha}+\left((-1)^{p-1} \prod_{i=0}^{p-1} B_{i}\right) y_{-1}^{\alpha}}{1+(-1)^{p-1} \prod_{i=0}^{p-1} B_{i}}
$$

and so for $\alpha=0, \ldots, p-1$

$$
\lim _{n \rightarrow \infty} x_{p n+p-1+\alpha}=\frac{x_{p-1+\alpha}+(-1)^{p-1} \prod_{i=0}^{p-1} B_{i} x_{-1+\alpha}}{1+(-1)^{p-1} \prod_{i=0}^{p-1} B_{i}} .
$$

In view of Equation (17) with $D_{n}=0, n=0,1, \ldots$, we have that for $\alpha=2,3, \ldots, p-1$

$$
x_{p-1+\alpha}=\left(1+\sum_{j=0}^{p-2+\alpha}(-1)^{j+1} \prod_{i=0}^{j} B_{i}\right) x_{0}+\left(\sum_{j=0}^{p-2+\alpha}(-1)^{j} \prod_{i=0}^{j} B_{i}\right) x_{-1}
$$

and

$$
x_{\alpha-1}=\left(1+\sum_{j=0}^{\alpha-2}(-1)^{j+1} \prod_{i=0}^{j} B_{i}\right) x_{0}+\left(\sum_{j=0}^{\alpha-2}(-1)^{j} \prod_{i=0}^{j} B_{i}\right) x_{-1} .
$$

Then for $\alpha=2,3, \ldots, p-1$ and after simplifying terms we obtain

$$
x_{p-1+\alpha}+\left((-1)^{p-1} \prod_{i=0}^{p-1} B_{i}\right) x_{\alpha-1}=\left(1+\sum_{j=0}^{p-2}(-1)^{j+1} \prod_{i=0}^{j} B_{i}\right) x_{0}+\left(\sum_{j=0}^{p-1}(-1)^{j} \prod_{i=0}^{j} B_{i}\right) x_{-1} .
$$

It should be noticed that by using Equation (37) and the initial conditions we also get Equation (38) for the cases $\alpha=0,1$. Thus for $\alpha=0,1, \ldots, p-1$

$$
\lim _{n \rightarrow \infty} x_{p n+p-1+\alpha}=\frac{\left(1+\sum_{j=0}^{p-2}(-1)^{j+1} \prod_{i=0}^{j} B_{i}\right) x_{0}+\left(\sum_{j=0}^{p-1}(-1)^{j} \prod_{i=0}^{j} B_{i}\right) x_{-1}}{1+(-1)^{p-1} \prod_{i=0}^{p-1} B_{i}} .
$$

Since all subsequences have the same limit, we finally obtain the formula for the limit.

Example 7. Consider the difference equation

$$
x_{n+1}=\left(1-y_{n+1}\right) x_{n}+y_{n+1} x_{n-1}, \quad n=0,1, \ldots
$$

where

$$
y_{n+1}=\frac{1+y_{n}}{y_{n-1}}, \quad n=0,1, \ldots
$$

Assume that $y_{-1}, y_{0} \notin\left\{-1,0, \frac{1 \pm \sqrt{5}}{2}\right\}$ and $y_{-1}+y_{0}+1 \neq 0$. Then the solution $\left\{y_{n}\right\}$ of Equation (40) is periodic of period 5 , that is the solution is

$$
\ldots, y_{-1}, y_{0}, \frac{1+y_{0}}{y_{-1}}, \frac{1+y_{0}+y_{-1}}{y_{0} y_{-1}}, \frac{1+y_{-1}}{y_{0}}, y_{-1}, y_{0}, \ldots
$$

Equation (40), see [12] also has the invariant

$$
I_{n}=\left(1+\frac{1}{y_{n-1}}\right)\left(1+\frac{1}{y_{n}}\right)\left(1+y_{n}+y_{n-1}\right), \quad n=0,1, \ldots
$$


and so for $n \geq 0$

$$
I_{n}=I_{0}=\left(1+\frac{1}{y_{-1}}\right)\left(1+\frac{1}{y_{0}}\right)\left(1+y_{0}+y_{-1}\right)=y_{1} y_{2} y_{3} y_{-1} y_{0} .
$$

Thus from Corollary 2 we obtain the following:

(1) If $I_{0} \leq-1$ or $I_{0}>1$, then every nonconstant solution of Equation (39) is unbounded.

(2) If $I_{0}=1$, then every nonconstant solution of Equation (39) is of period 10.

(3) If $\left|I_{0}\right|<1$, then every nonconstant solution of Equation (39) converges to a limit.

Suppose that $y_{-1}=-1 / 2, y_{0}=-1 / 4$, then the corresponding solution of Equation (40) is ..., $-3 / 2,2,-2,-1 / 2,-1 / 4, \ldots$, and so $I_{0}=3 / 4<1$. Thus every nonconstant solution of Equation (39) converges to a limit even though $g_{1}=y_{n+1}<1$ for some $n \geq 0$ and for other $n, g_{1}$ is strictly greater than 1 .

Remark 4. The same idea applies to Equation (15) with $D_{n}=0, n=0,1, \ldots$ For example, if there exists a constant $r$ such that $r<1$ and $0<\prod_{i=0}^{j} F_{i} \leq r^{j}, j=0,1, \ldots$, then the solutions of Equation (15) will converge to a limit even though there may be some $F_{i}>1$ for some $i=0, \ldots, j$. Since Equation (15) is used extensively to obtain our results, the behavior of the solutions of Equations (1), (17), (18) and (24) will depend on the product of the coefficients rather than the individual coefficients.

If for some $n, A_{n}=1, B_{n}=0$, then the solution of Equation (27) eventually becomes constant as the following result shows.

Theorem 3. Suppose that $A_{n}+B_{n}=1$ for all $n \geq 0$ and $A_{k}=1, B_{k}=0$ for some $k \geq 0$. Let $N$ be the first $k$ such that $B_{n}=0$. Then the solution $\left\{x_{n}\right\}$ of Equation (27) eventually becomes a constant. More precisely for $n \geq N$

$$
x_{n}=\left(1+\sum_{j=0}^{N-1}(-1)^{j+1} \prod_{i=0}^{j} B_{i}\right) x_{0}+\left(\sum_{j=0}^{N-1}(-1)^{j} \prod_{i=0}^{j} B_{i}\right) x_{-1} .
$$

Proof. Let $N \geq 0$ be the first $n$ such that $B_{n}=0$, that is $B_{N-1} \neq 0, B_{N}=0$. Then Equation (17) with $D_{n}=0, n \geq 0$ becomes for $N \geq 0$

$$
x_{N}=\left(1+\sum_{j=0}^{N-1}(-1)^{j+1} \prod_{i=0}^{j} B_{i}\right) x_{0}+\left(\sum_{j=0}^{N-1}(-1)^{j} \prod_{i=0}^{j} B_{i}\right) x_{-1} .
$$

Note that if $N=0$, then $B_{0}=0$ and Equation (42) is satisfied. From Equation (42) we get that for $N \geq 0$

$$
\begin{aligned}
x_{N+1} & =\left(1+\sum_{j=0}^{N}(-1)^{j+1} \prod_{i=0}^{j} B_{i}\right) x_{0}+\left(\sum_{j=0}^{N}(-1)^{j} \prod_{i=0}^{j} B_{i}\right) x_{-1} \\
& =\left(1+\sum_{j=0}^{N-1}(-1)^{j+1} \prod_{i=0}^{j} B_{i}\right) x_{0}+\left(\sum_{j=0}^{N-1}(-1)^{j} \prod_{i=0}^{j} B_{i}\right) x_{-1}=x_{N} .
\end{aligned}
$$

Similarly we can show that $x_{n}=x_{N}, n \geq N$.

\section{Conclusions}

In this paper we consider the general nonautonomous second order difference Equation (1) where the functions $g_{i}: \mathbb{R}^{k} \rightarrow \mathbb{R}, i=0,1 ; k=1,2, \ldots$ satisfy condition (2). The condition (2) implies the existence of an invariant which then reduces Equation (1) to the first order nonautonomous difference equation such as Equation (13) which has closed form solution (14). Using the closed form solution we were able to obtain the global behavior of solutions of some nonautonomous second order difference equations, such as Equation (26). The different types of asymptotic dynamics that the general nonautonomous second order difference Equation (1) exhibits are either convergence to 
a constant or a to a period-two sequence, divergence to $\pm \infty$, or eventual equality to a constant or a period-two sequence. Some results are given for the case when the coefficients of Equation (1) are periodic sequences when one can reduce the dynamics to the autonomous case, but our technique here was based on explicit solutions formula, see Example 6. The presented results have potential to be extended to systems of difference equations.

Author Contributions: Both authors contributed equally in the writing of this article.

Conflicts of Interest: The authors declare no conflict of interest.

\section{References}

1. Janowski, E.; Kulenović, M.R.S. Attractivity and global stability for linearizable difference equations. Comput. Math. Appl. 2009, 57, 1592-1607. [CrossRef]

2. Janowski, E.; Kulenović, M.R.S.; Silić, E. Periodic Solutions of Linearizable Difference Equations. Int. J. Differ. Equ. 2011, 6, 113-125.

3. Bilgin, A.; Kulenović, M.R.S. Global Asymptotic Stability for Discrete Single Species Population Models. Discret. Dyn. Nat. Soc. 2017, 2017, 5963594 [CrossRef]

4. Sedaghat, H. Nonlinear Difference Equations, Theory with Applications to Social Science Models; Mathematical Modelling: Theory and Applications; Kluwer Academic Publishers: Dordrecht, The Netherlands, 2003; p. 15.

5. Sedaghat, H. Form Symmetries and Reduction of Order in Difference Equations; Advances in Discrete Mathematics and Applications; CRC Press: Boca Raton, FL, USA, 2011.

6. Elaydi, S. An Introduction to Difference Equations, 3rd ed.; Undergraduate Texts in Mathematics; Springer: New York, NY, USA, 2005; 539p.

7. Feuer, J.; Janowski, E.; Ladas, G. Invariants for some rational recursive sequences with periodic coefficients. J. Differ. Equ. Appl. 1996, 2, 167-174. [CrossRef]

8. Gibbons, C.; Kulenović, M.R.S.; Ladas, G.; Voulov, H.D. On the trichotomy character of $x_{n+1}=\left(\alpha+\beta x_{n}+\right.$ $\left.\gamma x_{n-1}\right) /\left(A+x_{n}\right)$. J. Differ. Equ. Appl. 2002, 8, 75-92. [CrossRef]

9. Kalabušić, S.; Kulenović, M.R.S. Rate of convergence of solutions of rational difference equation of second order. Adv. Differ. Equ. 2004, 1, 121-140. [CrossRef]

10. Kocic, V.L.; Ladas, G. Global Behavior of Nonlinear Difference Equations of Higher Order with Applications; Kluwer Academic Publishers: Dordrecht, The Netherlands, 1993.

11. Kulenović, M.R.S. Invariants and related Liapunov functions for difference equations. Appl. Math. Lett. 2000, 13, 1-8. [CrossRef]

12. Kulenović, M.R.S.; Ladas, G. Dynamics of Second Order Rational Difference Equations, with Open Problems and Conjectures; Chapman \& Hall/CRC Press: Boca Raton, FL, USA, 2001.

13. Kulenović, M.R.S.; Merino, O. Discrete Dynamical Systems and Difference Equations with Mathematica, Chapman and Hall/CRC: Boca Raton, FL, USA; London, UK, 2002.

14. Nesemann, T. Invariants and Liapunov functions for nonautonomous systems. Advances in difference equations, III. Comput. Math. Appl. 2001, 42, 385-392. [CrossRef]

(C) 2018 by the authors. Licensee MDPI, Basel, Switzerland. This article is an open access article distributed under the terms and conditions of the Creative Commons Attribution (CC BY) license (http://creativecommons.org/licenses/by/4.0/). 